\title{
Identification of serum biomarkers for aging and anabolic response
}

\author{
Camellia Banerjee ${ }^{1}$, Jagadish Ulloor ${ }^{2}$, Edgar L Dillon ${ }^{3}$, Qusai Dahodwala', Brittani Franklin¹, Thomas Storer², \\ Paola Sebastiani ${ }^{3}$, Melinda Sheffield-Moore ${ }^{4}$, Randall J Urban ${ }^{4}$, Shalender Bhasin ${ }^{2}$ and Monty Montano ${ }^{1^{*}}$
}

\begin{abstract}
Objective: With the progressive aging of the human population, there is an inexorable decline in muscle mass, strength and function. Anabolic supplementation with testosterone has been shown to effectively restore muscle mass in both young and elderly men. In this study, we were interested in identifying serum factors that change with age in two distinct age groups of healthy men, and whether these factors were affected by testosterone supplementation.
\end{abstract}

Methods: We measured the protein levels of a number of serum biomarkers using a combination of banked serum samples from older men (60 to 75 years) and younger men (ages 18 to 35), as well as new serum specimens obtained through collaboration. We compared baseline levels of all biomarkers between young and older men. In addition, we evaluated potential changes in these biomarker levels in association with testosterone dose (low dose defined as $125 \mathrm{mg}$ per week or below compared to high dose defined as $300 \mathrm{mg}$ per week or above) in our banked specimens.

Results: We identified nine serum biomarkers that differed between the young and older subjects. These ageassociated biomarkers included: insulin-like growth factor (IGF1), N-terminal propeptide of type III collagen (PIIINP), monokine induced by gamma interferon (MIG), epithelial-derived neutrophil-activating peptide 78 (ENA78), interleukin 7 (IL-7), p40 subunit of interleukin 12 (IL-12p40), macrophage inflammatory protein $1 \beta$ (MIP-1 $\beta$ ), platelet derived growth factor $\beta$ (PDGF $\beta$ ) and interferon-inducible protein 10 (IP-10). We further observed testosterone dose-associated changes in some but not all age related markers: IGF1, PIIINP, leptin, MIG and ENA78. Gains in lean mass were confirmed by dual energy X-ray absorptiometry (DEXA).

Conclusions: Results from this study suggest that there are potential phenotypic biomarkers in serum that can be associated with healthy aging and that some but not all of these biomarkers reflect gains in muscle mass upon testosterone administration.

Keywords: Testosterone, Age, Biomarker

\section{Introduction}

As the general population ages, there is an increased prevalence of loss in muscle mass, raising the risk for frailty, declines in functional mobility, and early mortality [1-4]. Loss of lean muscle can also be a comorbid condition in multiple chronic and acute disorders including cancer cachexia, HIV-associated weight loss, inflammatory sepsis, and age-associated sarcopenia

\footnotetext{
* Correspondence: mmontano@bu.edu

'Section of Infectious Diseases, Department of Medicine, Boston University

School of Medicine, 710 Albany Street, Boston MA, 02118, USA

Full list of author information is available at the end of the article
}

[5-8]. Biomarkers for healthy aging identifiable in the serum would be of substantial use in detecting age associated morbidities and initiating therapeutic pro-anabolic treatment.

Anabolic supplementation is broadly recognized to increase muscle mass in both elderly and young individuals $[9,10]$. Testosterone displays a dose dependent effect on gains of lean muscle mass and cross-sectional fiber area in both older and younger men [6,9,11-15]. However, because in some populations testosterone administration poses undesirable side effects, there is a motivation for identifying alternative, broadly effective

\section{Biomed Central}


anabolic therapeutics, such as selective androgen receptor modulators (SARMs) that improve muscle mass and physical function [16-18]. Therefore, the utility of serum biomarkers would help to gauge pro-anabolic activity of SARMs and therefore be of substantial value in clinical research to ameliorate declines in muscle mass and function.

In this study we were interested in identifying ageassociated biomarkers for healthy aging and evaluating whether biomarkers that differ between healthy young and older men at baseline, also differ in response to graded doses of testosterone. To achieve this, we used banked serum specimens from younger and older men to measure selected soluble cytokines and growth factors, based on predicted biomarkers from previous studies by us and others [2,15,19-25]. Herein, we report results from this pilot analysis to identify biomarkers that change either in association with age and/or testosterone dosage.

\section{Methods}

\section{Sample Population (Boston, MA)}

The samples used for this study were obtained from a previously reported double-blind, randomized study that consisted of a 4-week control period, 20-week treatment period and 16-week recovery period [11,13]. Participants included sixty young men (age range 18 to 35 years) and sixty-one older men (age range 60 to 75 years). All subjects provided informed written consent according to protocol approved by the Charles Drew University and Research and Education Institute. Exclusion criteria included 1) presence of prostate disease defined as cancer, an American Urological Association symptom score of greater than 7, a prostate-specific antigen level greater than $4 \mathrm{ng} / \mathrm{ml}, 2$ ) hematocrit above $48 \%, 3$ ) diabetes mellitus, 4) heart problems including myocardial infarction or congestive heart failure all measured using a 12-lead electrocardiogram monitoring to exclude symptoms present during exercise as well, 5) severe sleep apena, 6) administration of androgenic steroids in the past year, 7) participation in sports events, resistance training or moderate to heavy endurance exercise training and 8) baseline testosterone levels below $300 \mathrm{ng} / \mathrm{dL}$. For more in depth description of enrollment criteria and physical function, see Bhasin et al, in [11,13]. Stored serum samples at baseline and after treatment were used from 20 of the younger men and 19 of the older men based on availability. Mean baseline testosterone levels for younger men were $586 \mathrm{ng} / \mathrm{dL}$ and $358 \mathrm{ng} / \mathrm{dL}$ for older men.

\section{Sample Population (Houston, TX)}

Stored baseline serum samples were used from 20 older men (age range 60 to $85 \mathrm{yrs}$ ) recruited through the
Sealy Center of Aging Volunteer Registry at the University of Texas Medical Branch (UTMB) in Galveston, TX for inclusion in a randomized double-blinded placebocontrolled testosterone intervention study. All subjects provided informed written consent according to the guidelines established by the UTMB institutional review board and were medically screened. Qualified subjects had endogenous testosterone concentrations below 500 $\mathrm{ng} / \mathrm{dL}$ and were otherwise healthy. To assess medical eligibility, subjects underwent a battery of tests including a history and physical examination, complete blood count, metabolic panel including fasting serum glucose and insulin, an electrocardiogram (ECG), plasma electrolytes, prostate specific antigen (PSA), liver and renal function and lipid panel. Subjects were included based upon their ability to provide regular transportation to the Clinical Research Center (CRC) at UTMB. Subject exclusion criteria included the following: 1) serum testosterone $>500 \mathrm{ng} / \mathrm{dL}, 2$ ) indication of cardiovascular disease or heart problems assessed via a resting ECG and a Bruce protocol exercise stress test, 3) previous history of angina or myocardial infarction, 4) PSA $>4.0$ $\mu \mathrm{g} / \mathrm{L}, 5)$ history of prostate cancer, 6) history of severe benign prostatic hypertrophy, 7) LDL > $200 \mathrm{mg} / \mathrm{dL}, 8$ ) hematocrit > 51\%, 9) hypertension (>140/90 $\mathrm{mmHg}$ ), 10) BMI $>35,11$ ) history of hepatitis or $3 \times$ elevation of Alk phos, ALT, AST, 12) illnesses including diabetes, cancer, COPD, sleep apnea or any other causing disability, 13) bone related disorders, 14) DEXA lumbar score $>-2.5,15)$ currently taking Coumadin, glucocorticoids, androgens, or anti-bone-resorptive agents, and 16) regular physical exercise. These inclusion/exclusion criteria reflect those recommended by the Clinical Guidelines Subcommittee Task Force of The Endocrine Society[26] and previously published trials with testosterone and older men $[27,28]$. Mean baseline testosterone levels for these older men were $320 \mathrm{ng} / \mathrm{dL}$.

\section{Testosterone supplementation (Boston, MA)}

Serum samples were obtained from men who participated in a randomized testosterone supplementation trial. Men were treated with monthly injections of a long-acting $\mathrm{GnRH}$ agonist (Lupron depot, $7.5 \mathrm{mg}$; TAP, North Chicago, IL) to suppress endogenous testosterone production, and concomitantly weekly injections of one of five doses of testosterone enanthate (Delastryl, Savient Pharmaceuticals, NJ) [11]. Based on dichotomous functional outcomes in previous reports, testosterone doses were categorized as low (i.e., $25 \mathrm{mg}, 50 \mathrm{mg}$, and $125 \mathrm{mg}$ ) and high (i.e., $300 \mathrm{mg}$ and $600 \mathrm{mg}$ ).

\section{Biomarker measurements}

The serum specimens were selected based on quality and availability. Quality was determined by visual 
inspection. Serum specimens for both populations had been collected, centrifuged and stored at similar conditions in both places. Serum factors were measured at two time intervals: early in the study, i.e., at baseline or within the first two weeks of starting $\mathrm{GnRH}$ and testosterone treatment, and later in the study, i.e., twenty weeks after initiation of $\mathrm{GnRH}$ and testosterone treatment. Insulin-like growth factor I (IGF1) was measured using an enzyme-linked immunosorbent assay (ELISA) using a non-extraction IGF-1 ELISA kit (Diagnostic Systems Laboratories, TX) in both young and old at baseline and after treatment. Pro-collagen III N-terminal peptide (PIIINP) was measured using validated equilibrium radioimmunoassay (RIA) (Orion Diagnostics, Espoo, Finland) as described previously[15,29] in both the young and the old subjects at baseline and after testosterone supplementation.

The remaining serum factors were measured using a multiplex Luminex platform (Panomics, Fremont, CA) as described previously [30]. This assay uses xMAP technology, a multi-analyte profiling Luminex technology, to detect and quantify multiple protein targets. The samples were run on a LiquiChip (Qiagen) and were analyzed using Qiagen Liquichip Analyzer software (Version 1.0.5.17455). A 35-plex was run on serum from the young men measuring ENA78, Eotaxin, FGF Basic, G-CSF, GMCSF, GRO- $\alpha$, IFN $\gamma$, IL1 $\alpha$, IL1 $\beta$, IL-10, IL-12(p40), IL-12 (p70), IL-13, IL-15, IL-17, IL-17F, IL-1RA, IL-2, IL-4, IL-5, IL-6, IL-7, IL-8, IP10, Leptin, MCP-3, MIG, MIP1 $\alpha$, MIP1 $\beta$, NGF, PDGF-BB, RANTES, TNF $\alpha$ and TNF $\beta$ at baseline and after testosterone treatment. For those biomarkers that showed a change with testosterone treatment in the young, another 3-plex was run on serum from the older men treated with testosterone at baseline and after treatment measuring leptin, MIG and ENA78. A 30-plex assay was run to measure baseline cytokine levels of a separate group of older men before treatment measuring eotaxin, FGF basic, GCSF, GM-CSF, GRO $\alpha$, IFN $\gamma$, IL1 $\alpha$, IL1 $\beta$, IL-10, IL-12(p40), IL-12(p70), IL-13, IL-17A, IL-2, IL-4, IL-5, IL-6, IL-7, IL-8. IP-10, MCP-1, MCP-3, MIP$1 \alpha$, MIP-1 $\beta$, NGF, PDGF-BB, RANTES, TNF $\alpha$, TNF $\beta$, and VEGF. Values outside of the range of the standard curve were omitted for the multiplex assays. The lower limit of detection for these analytes was $1 \mathrm{pg} / \mathrm{ml}$. The CV range for inter-assay variability for the analytes was $6.73 \%$ $-17.25 \%$, with an average of $12.24 \%$.

\section{Statistical Methods}

Baseline values of biomarkers were compared between younger and older men using a parametric two-sample ttest assuming unequal variances and a non-parametric two-sample Wilcoxon rank-sum test giving similar pvalues. The determination of significance in biomarker response was based on a matched pair analysis of early versus late levels, and a bivariate categorical analysis of testosterone dose (low versus high) and age (younger men versus older men), using a parametric two-sample t-test with unequal variances and a non-parametric two-sample Wilcoxon rank-sum test with similar results. Statistical analyses were carried out in STATA version 8.0 (Stata Corp, College Station, Tex) and JMP 8.0.2 (SAS Institute Inc, Cary, NC). Values shown are all displayed as the mean plus/minus standard deviation unless otherwise indicated. Box plots are shown as quantiles, with the median (line in box), quartile range (edges of box), and extremes (vertical lines and points). In single-plex assays, siginificance was set at $\mathrm{p}<0.05$. In multiplex assays, a $\mathrm{p}$-value $<0.05$ was used to denote statististical significance while a p-value $<0.05 /$ $10=0.005$ was used to take into account multiple comparisons using Bonferrorni correction.

\section{Results}

\section{Study Population Characteristics}

The study population consisted of healthy younger men (18-35 years old) and healthy older men (60-75 years old). We screened selected serum chemokines, cytokines, growth factors and angiogenic factors that we expected to change with age and possibly testosterone administration based on previous studies of aging and anabolic supplementation $[2,15,19-24]$. Serum samples were screened within the first two weeks of testosterone treatment and at week 20, the end of treatment period, to look for changes associated with testosterone dosage. Summary characteristics of the sampled population of younger and older men used for our serum data are shown in Table 1.

\section{Age related serum profile}

The results of our multiplex assay identify nine serum proteins that differed (significance $\mathrm{p}<0.005$ ) between

Table 1 Characteristics of study population

\begin{tabular}{llll}
\hline & Young & Old & $\begin{array}{l}\text { P } \\
\text { value }\end{array}$ \\
\hline Age $(\mathbf{y r})$ & $27 \pm 5(53)$ & $68 \pm 7(39)$ & $<0.001$ \\
Height $(\mathbf{c m})$ & $175 \pm 7(53)$ & $175 \pm 7(39)$ & 0.9683 \\
Weight $(\mathbf{k g})$ & $74.9 \pm 10.0$ & $84.3 \pm 13.1$ & 0.0003 \\
& $(53)$ & $(39)$ & \\
Body mass index (\%) & $24 \pm 3(53)$ & $27 \pm 4(39)$ & $<0.001$ \\
Serum total testosterone & $586 \pm 190$ & $339 \pm 95(39)$ & $<0.001$ \\
(ng/dl) & $(53)$ & & \\
Population Houston, TX & N/A & $358 \pm 86(20)$ & N/A \\
Population Boston, MA & $586 \pm 190$ & $320 \pm 103$ & N/A \\
& $(53)$ & $(19)$ & \\
Lean Body Mass (mg/cm ${ }^{2}$ ) & $57336 \pm 7209$ & $58545 \pm 7033$ & 0.4245 \\
& $(52)$ & $(39)$ & \\
\hline
\end{tabular}

Baseline characteristics of subjects evaluated in this study are shown. The number of subject used for each baseline value is indicated in parenthesis. The values are displayed as plus/minus standard deviation. The $p$-values are based on a Student $t$-test. 
the two age groups, as shown in Figure 1. Eight of the nine serum proteins decreased with age: IGF-1, PIIINP, MIP-1 $\beta$, IP-10, IL-7, IL-12p40, PDGF $\beta$ and eotaxin. The ninth serum protein, MIG showed higher levels in the older men relative to the younger men. All other analytes were below detection or not significant. These pilot data suggest that serum profiling may be a useful strategy to gauge healthy aging. However, because these data only use measurements at two age intervals, it remains unclear whether these biomarkers change monotonically with age. To address the relationship between each of the serum biomarkers we measured the Pearson's correlation, as shown in Table 2. Six biomarkers (MIP-1 $\beta$, IP-10, IL-7, IL-12p40, PDGF $\beta$ and eotaxin) displayed robust $r^{2}$ values $\geq 0.75$. To evaluate potential networks between these biomarkers, we utilized Ingenuity Pathway analysis (IPA) and observed that Akt, NFkB and TGF $\beta$ signaling were common pathways among five of these biomarkers, MIP-1 $\beta$, IP-10, IL7, IL-12p40, PDGF $\beta$ (data not shown), possibly suggesting that growth, inflammatory and fibrotic regulatory mechanisms, respectively contribute to the biomarker profile.

\section{Testosterone related response in serum biomarkers}

Testosterone supplementation has been shown to increase muscle mass in both young and older men. We were interested in seeing if the factors affected by age were also responsive to testosterone supplementation and in finding markers for testosterone supplementation that are robust in both the young and the old. We evaluated the response to graded doses of testosterone in relation to a young age group (Figure 2), an older age group (Figure 3 ) as well as both groups combined (Figure 4 ). Due to the small sample size, we based our analysis on two groups, low dosage ( $25 \mathrm{mg}$ per week to 125 $\mathrm{mg}$ per week) and high dosage (greater than $300 \mathrm{mg}$ per week) of testosterone, looking at the change in biomarker levels between the baseline and the end of treatment. Based on this approach, we observed three serum factors measured in single-plex assays showed a significant change in association with testosterone dose in the young, with significance values of $\mathrm{p}<0.05$ for each independent assay. These include PIIINP, leptin, and IGF-1 (Figure 2). We also observed a significant difference with testosterone dosage in levels of ENA78 and MIG, however due to a small sample size $(n=2)$, these

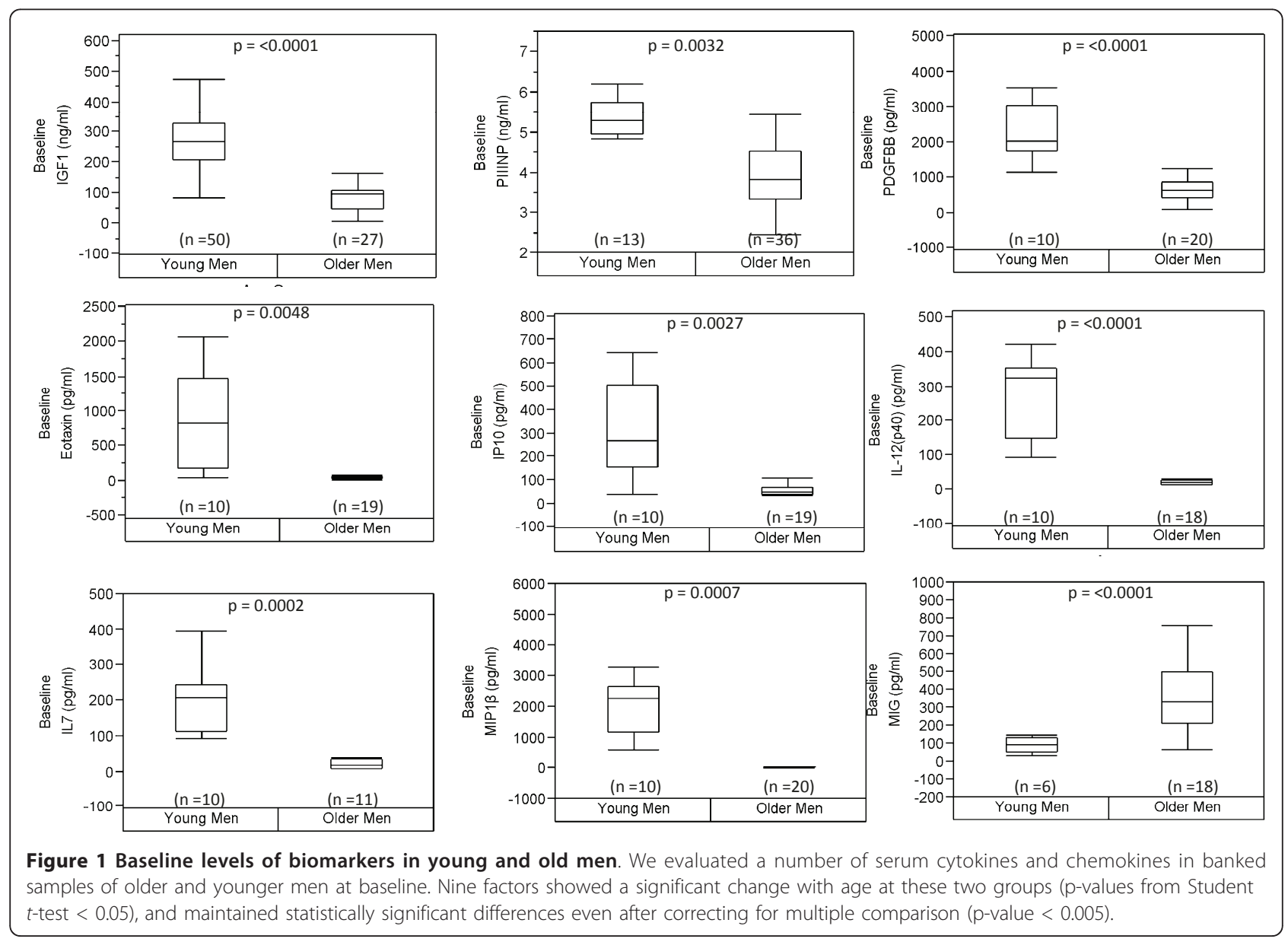


Table 2 Correlation between Baseline Markers.

\begin{tabular}{|c|c|c|c|c|c|c|c|c|c|c|c|c|}
\hline & Leptin & IL5 & MCP1 & VEGF & PIIINP & PDGFBB & IGF1 & Eotaxin & IL7 & IP10 & MIP1 $\beta$ & IL12(p40) \\
\hline Leptin & & 0.50 & -0.28 & 0.09 & 0.16 & 0.10 & -0.26 & 0.02 & 0.11 & -0.06 & -0.11 & -0.15 \\
\hline IL5 & 0.50 & & 0.42 & 0.50 & 0.04 & 0.01 & -0.23 & 0.00 & 0.09 & 0.02 & -0.15 & -0.18 \\
\hline MCP1 & -0.28 & 0.42 & & -0.04 & 0.10 & -0.07 & -0.57 & -0.30 & -0.04 & 0.65 & 0.54 & -0.06 \\
\hline VEGF & 0.09 & 0.50 & -0.04 & & -0.34 & 0.51 & 0.29 & 0.50 & 0.35 & 0.34 & 0.14 & -0.43 \\
\hline PIIINP & 0.16 & 0.04 & 0.10 & -0.34 & & 0.46 & 0.34 & 0.44 & 0.57 & 0.71 & 0.43 & 0.62 \\
\hline PDGFBB & 0.10 & 0.01 & -0.07 & 0.51 & 0.46 & & 0.62 & 0.68 & 0.85 & 0.79 & 0.82 & 0.85 \\
\hline IGF1 & -0.26 & -0.23 & -0.57 & 0.29 & 0.34 & 0.62 & & 0.43 & 0.54 & 0.52 & 0.62 & 0.69 \\
\hline Eotaxin & 0.02 & 0.00 & -0.30 & 0.50 & 0.44 & 0.68 & 0.43 & & 0.75 & 0.82 & 0.64 & 0.69 \\
\hline IL7 & 0.11 & 0.09 & -0.04 & 0.35 & 0.57 & 0.85 & 0.54 & 0.75 & & 0.69 & 0.67 & 0.65 \\
\hline IP10 & -0.06 & 0.02 & 0.65 & 0.34 & 0.71 & 0.79 & 0.52 & 0.82 & 0.69 & & 0.73 & 0.80 \\
\hline MIP1 $\beta$ & -0.11 & -0.15 & 0.54 & 0.14 & 0.43 & 0.82 & 0.62 & 0.64 & 0.67 & 0.73 & & 0.89 \\
\hline IL-12(p40) & -0.15 & -0.18 & -0.06 & -0.43 & 0.62 & 0.85 & 0.69 & 0.69 & 0.65 & 0.80 & 0.89 & \\
\hline
\end{tabular}

A pairwise Pearson's correlation was done to evaluate the relationship between biomarker levels in the young and old subjects combined. The $r^{2}$ values for each pairwise correlation is shown. The $r^{2}$ values $\geq 0.75$ were used to identify networks using Ingenuity Pathway Analysis (IPA). In data not shown Akt, NF $\kappa$ B and TGF $\beta$ pathways were common among these biomarkers.

data are not shown. In the older men, only leptin and DEXA displayed a similar trend change as seen in the young men at a $\mathrm{p}$-value of $\leq 0.05$ for each independent assay (Figure 3). Notably, IGF1 and PIIINP showed a trend change but did not reach significance. This may be due to the small sample size of the older population and/or the sampling time of the baseline values (within first two weeks) in the young, since these biomarkers may have already begun to rise and thereby affect the change observed. When the data from older men and younger men were combined, we observed that three of the biomarkers seen in the young, i.e., IGF1, PIIINP and leptin, as well as DEXA, remained significant using a pvalue of $\leq 0.05$ for each independent assay (Figure 4). These data indicate that a subset of biomarkers associated with healthy aging men also change with testosterone administration, given the limits of detection for the assays used in this study. Whether a similar profile
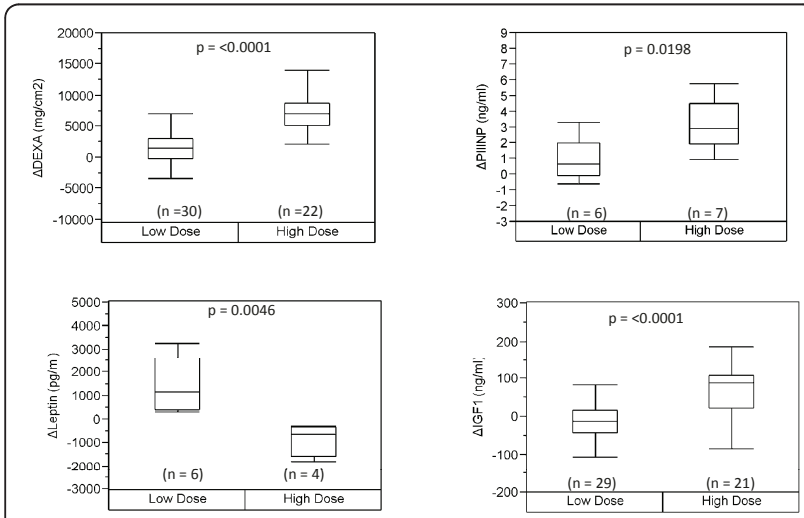

Figure 2 Biomarker response based on testosterone dose in the young. We evaluated the change in biomarkers that differ significantly between low and higher doses of testosterone in younger subjects. P-values are indicated based on a Student $t$-test. of anabolic change occurs with exercise or physical function improvements remains an important question in efforts to more fully understand the limits of androgen improvements and specificity. These data also represent the first comparative evaluation of biomarkers in young and old subjects receiving equivalent testosterone supplementation.

\section{Discussion}

In this pilot study we describe serum proteins that change in association with healthy aging among men between the ages of 18 and 35 compared with older men between the ages of 60 and 85 . We provide evidence that a subset of these biomarkers, but not all, appear to be responsive to anabolic administration. These data support the high likelihood of a serum
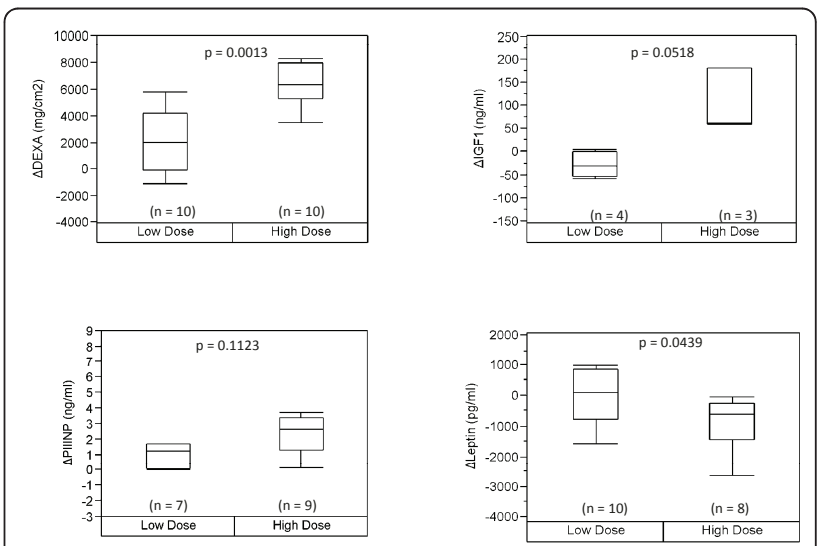

Figure 3 Biomarker response based on testosterone dose in the older group. We evaluated the change in biomarkers that differ significantly between low and higher doses of testosterone in older subjects. Leptin was found to be significant to a p-value of $<$ 0.05, however both PIIINP and IGF1 showed a trend change. Pvalues are indicated based on a Student $t$-test. 

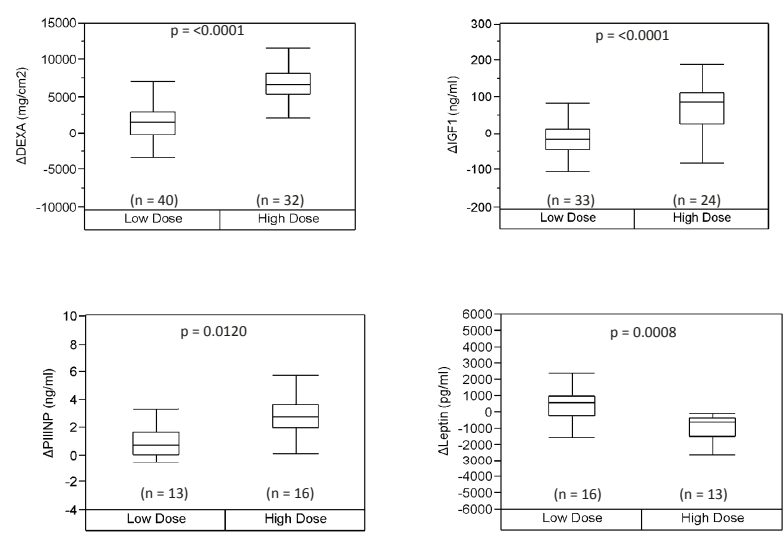

Figure 4 Biomarker response based on testosterone dose in the young and old combined. We evaluated the change in biomarkers that differ significantly between low and higher dose of testosterone in combined young and older subjects. Three factors in addition to DEXA were found to be significant to $p$-value less than 0.05. P-values are indicated based on a Student $t$-test.

profile for aging that may be useful for establishing response profiles for therapeutic androgen administration in older and younger men, particularly when measured against a reliable age reference group for baseline biomarker levels. Thus, the use of biomarkers in evaluating anabolic response would benefit from studies that precisely define the profile of biomarker change during healthy aging to adequately gauge the effect(s) of gains associated with anabolic therapy.

Of the serum biomarkers screened at baseline in both healthy young and older men, we provide evidence for nine that displayed differences in baseline levels at two different age groups: IGF1, PDGFBB, PIIINP, eotaxin, IP-10, IL-12(p40), IL-7, MIP1 $\beta$ and MIG. IGF1 levels, have been shown previously to decrease with increased age and have been associated with muscle declines [31]. PIIINP, a marker of remodeling and collagen synthesis has not been previously studied with aging. The declines in PIIINP levels in older men suggest a possible decrease in remodeling with age. The remaining immunoregulatory biomarkers collectively suggest a possible decrease in T-cell and neutrophil response with healthy aging that could influence muscle maintenance with aging [32-38]. Pathway analysis using IPA for signaling networks common to these biomarkers highlight a potential role for Akt, NF $\kappa$ and TGF $\beta$ pathways, all of which have been independently associated with aging [39-42]. The correlated changes in these biomarkers suggest that perhaps a change in one of these markers may be predictive of changes in other networked biomarkers as an aggregate. However, this possibility of aggregate biomarker profiles will require further study with larger sampling than was available in this pilot study.
Previous studies on the effects of testosterone supplementation have indicated that similar gains in muscle strength and fat free mass are observed in older men compared with younger men $[11,13]$. Previous separate studies have addressed individual serum biomarkers that change in association with anabolic administration (e.g., leptin, IGF-1) in the young [11,22] and old [23,27]. Importantly, our study confirms a recent study showing that PIIINP levels increase with testosterone and predict gains in lean muscle mass in both young men and older men [15]. Although we do confirm concordant gains with some biomarkers, we also notice that despite similar gains in muscle strength and mass, older men differed from younger men in baseline levels of biomarkers and in the response profile of these markers associated with testosterone supplementation. This suggests, at least for this limited set of biomarkers, and in these subjects, that gains in muscle mass and potentially associated gains in physical function do not display simple concordance with the serum profile. This may suggest the existence of complex mechanisms for testosterone response that will require further study. Additional biomarkers that accurately reflect anabolic response would assist in more accurately defining anabolic therapeutic outcomes as well as other approaches for gains in muscle mass and function, e.g., exercise and other function promoting therapeutics. The direct and combined comparative study described in this report validates the robustness and non-equivalency of multiple markers in association with dose and age - an observation that has not been fully recognized to date.

Finally, previous studies have shown that testosterone levels generally decline with age in men $[17,43]$. Whether there is a feedback relationship between age dependent declines in testosterone and biomarkers is beyond the scope of this study but remains an intriguing possibility. The dampened biomarker profile observed with an older age group compared to a younger age group in this study suggests that testosterone's effect on anabolic pathways may change with age while nevertheless achieving the same gains in mass and strength. Future studies evaluating age- and anabolic-dependent shifts in biomarker levels will assist in identifying novel pathways that can be used to promote gains in muscle mass across a broad age range.

\section{Acknowledgements}

The authors wish to acknowledge support from the Boston OAIC Pepper Center and NIAMS R01 AR055115 (MM). This work was supported in part by the UTMB Claude D. Pepper Older Americans Independence Center NIH/NIA Grant \# P30 AG024832 and also supported in part by the UTMB Institute for Translational Sciences - Clinical Research Center (ITS-CRC) grant 1UL1RR029876-01 from the National Center for Research Resources, National Institutes of Health.

\section{Author details}

${ }^{1}$ Section of Infectious Diseases, Department of Medicine, Boston University School of Medicine, 710 Albany Street, Boston MA, 02118, USA. ${ }^{2}$ Section of 
Endocrinology, Diabetes and Nutrition, Department of Medicine, Boston University School of Medicine, 710 Albany Street, Boston MA, 02118, USA ${ }^{3}$ Department of Biostatistics, Boston University School of Public Health, 715 Albany Street, Boston, 02118 , USA. ${ }^{4}$ Division of Endocrinology and Metabolism, Department of Medicine, University of Texas Medical Branch, 301 University Boulevard, Galveston TX, 77555, USA.

\section{Authors' contributions}

CB drafted the manuscript, ran assays and analyzed the data. JU ran the RIA and helped analyze data for PIIINP experiments. ED, MSM and RJU participated in the design and coordination of the questions asked using the Texas cohort. QD and BF ran assays and drafted figures and tables. TS and SB conceived concept and provided samples and data for the Boston cohort. PS provided statistical analysis. MM conceived the questions asked overall in this study and participated in the design, coordination and writing of the manuscript. All authors have read and approved this manuscript.

\section{Competing interests}

The authors declare that they have no competing interests.

Received: 16 February 2011 Accepted: 20 June 2011

Published: 20 June 2011

\section{References}

1. Yarasheski KE: Exercise, aging, and muscle protein metabolism. J Gerontol A Biol Sci Med Sci 2003, 58(10):M918-22.

2. Peake JM, Della Gatta P, Cameron-Smith D: Aging and its effects on inflammation in skeletal muscle at rest and following exercise-induced muscle injury. Am J Physiol Regul Integr Comp Physiol 2010, 298(6): R1485-95.

3. Rantanen T, et al: Muscle strength and body mass index as long-term predictors of mortality in initially healthy men. J Gerontol A Biol Sci Med Sci 2000, 55(3):M168-73.

4. Janssen I: Influence of sarcopenia on the development of physical disability: the Cardiovascular Health Study. J Am Geriatr Soc 2006, 54(1):56-62.

5. Dudgeon WD, et al: Counteracting muscle wasting in HIV-infected individuals. HIV Med 2006, 7(5):299-310.

6. Lynch GS, Schertzer JD, Ryall JG: Therapeutic approaches for muscle wasting disorders. Pharmacol Ther 2007, 113(3):461-87.

7. Filippatos GS, Anker SD, Kremastinos DT: Pathophysiology of peripheral muscle wasting in cardiac cachexia. Curr Opin Clin Nutr Metab Care 2005, 8(3):249-54.

8. Strassburg S, Springer J, Anker SD: Muscle wasting in cardiac cachexia. Int J Biochem Cell Biol 2005, 37(10):1938-47.

9. Bhasin $\mathrm{S}$, et al: Testosterone replacement and resistance exercise in HIV infected men with weight loss and low testosterone levels. JAMA 2000, 283(6):763-70

10. Jenkins MW, et al: Phenotyping transgenic embryonic murine hearts using optical coherence tomography. Appl Opt 2007, 46(10):1776-81

11. Bhasin $\mathrm{S}$, et al: Testosterone dose-response relationships in healthy young men. Am J Physiol Endocrinol Metab 2001, 281(6):E1172-81.

12. Sinha-Hikim I, et al: Testosterone-induced increase in muscle size in healthy young men is associated with muscle fiber hypertrophy. Am J Physiol Endocrinol Metab 2002, 283(1):E154-64.

13. Bhasin $\mathrm{S}$, et al: Older men are as responsive as young men to the anabolic effects of graded doses of testosterone on the skeletal muscle. J Clin Endocrinol Metab 2005, 90(2):678-88.

14. Singh $A B$, et al: The effects of varying doses of $T$ on insulin sensitivity, plasma lipids, apolipoproteins, and C-reactive protein in healthy young men. J Clin Endocrinol Metab 2002, 87(1):136-43.

15. Bhasin S, et al: N-terminal propeptide of type III procollagen as a biomarker of anabolic response to recombinant human $\mathrm{GH}$ and testosterone. J Clin Endocrinol Metab 2009, 94(11):4224-33.

16. Bhasin $\mathrm{S}$, et al: Drug insight: Testosterone and selective androgen receptor modulators as anabolic therapies for chronic illness and aging. Nat Clin Pract Endocrinol Metab 2006, 2(3):146-59.

17. Gooren $\perp$ : Androgens and male aging: Current evidence of safety and efficacy. Asian J Androl 12(2):136-51.

18. Basaria $\mathrm{S}$, et al: Adverse events associated with testosterone administration. N Engl J Med 363(2):109-22.
19. Rommel C, et al: Mediation of IGF-1-induced skeletal myotube hypertrophy by $\mathrm{PI}(3) \mathrm{K} / \mathrm{Akt} / \mathrm{mTOR}$ and $\mathrm{PI}(3) \mathrm{K} / \mathrm{Akt} / \mathrm{GSK} 3$ pathways. Nat Cell Biol 2001, 3(11):1009-13.

20. Stitt TN, et al: The IGF-1/PI3K/Akt pathway prevents expression of muscle atrophy-induced ubiquitin ligases by inhibiting FOXO transcription factors. Mol Cell 2004, 14(3):395-403.

21. Friedman JM: The function of leptin in nutrition, weight, and physiology. Nutr Rev 2002, 60(10 Pt 2):S1-14, discussion S68-84, 85-7.

22. Hislop MS, et al: Effects of anabolic-androgenic steroid use or gonadal testosterone suppression on serum leptin concentration in men. Eur J Endocrinol 1999, 141(1):40-6.

23. Sih $\mathrm{R}$, et al: Testosterone replacement in older hypogonadal men: a 12month randomized controlled trial. J Clin Endocrinol Metab 1997, 82(6):1661-7.

24. Schaap $L A$, et al: Inflammatory markers and loss of muscle mass (sarcopenia) and strength. Am J Med 2006, 119(6):526 e9-17.

25. Montano $\mathrm{M}$, et al: Transcriptional profiling of testosterone-regulated genes in the skeletal muscle of human immunodeficiency virus-infected men experiencing weight loss. J Clin Endocrinol Metab 2007, 92(7):2793-802.

26. Committee on Assessing the Need for Clinical Trials of Testosterone Replacement Therapy, Executive Summary. In Testosterone and aging: clinical research directions. Edited by: Liverman C, Blazer D. Washington, DC; 2004:1-10.

27. Ferrando $A A$, et al: Testosterone administration to older men improves muscle function: molecular and physiological mechanisms. Am J Physiol Endocrinol Metab 2002, 282(3):E601-7.

28. Urban RJ, et al: Testosterone administration to elderly men increases skeletal muscle strength and protein synthesis. Am J Physiol 1995, 269(5 Pt 1):E820-6

29. Risteli J, et al: Rapid equilibrium radioimmunoassay for the aminoterminal propeptide of human type III procollagen. Clin Chem 1988, 34(4):715-8.

30. Shurin GV, et al: Dynamic alteration of soluble serum biomarkers in healthy aging. Cytokine 2007, 39(2):123-9.

31. Perrini $\mathrm{S}$, et al: The GH/IGF1 axis and signaling pathways in the muscle and bone: mechanisms underlying age-related skeletal muscle wasting and osteoporosis. J Endocrinol 205(3):201-10.

32. Kim HR, et al: Altered IL-7Ralpha expression with aging and the potential implications of IL-7 therapy on CD8+ T-cell immune responses. Blood 2006, 107(7):2855-62.

33. Phillips JA, et al: IL-7 gene therapy in aging restores early thymopoiesis without reversing involution. J Immunol 2004, 173(8):4867-74.

34. Andrew $D$, Aspinall R: Age-associated thymic atrophy is linked to a decline in IL-7 production. Exp Gerontol 2002, 37(2-3):455-63.

35. Cooper AM, Khader SA: IL-12p40: an inherently agonistic cytokine. Trends Immunol 2007, 28(1):33-8.

36. Yoshikawa $\mathrm{H}$, et al: Elevation of IL-12 p40 and its antibody in myasthenia gravis with thymoma. J Neuroimmunol 2006, 175(1-2):169-75.

37. Tanaka Y, et al: T-cell adhesion induced by proteoglycan-immobilized cytokine MIP-1 beta. Nature 1993, 361(6407):79-82.

38. Swift $\mathrm{ME}$, et al: Age-related alterations in the inflammatory response to dermal injury. J Invest Dermatol 2001, 117(5):1027-35.

39. Adler AS, et al: Motif module map reveals enforcement of aging by continual NF-kappaB activity. Genes Dev 2007, 21(24):3244-57.

40. Carlson ME, et al: Relative roles of TGF-beta1 and Wnt in the systemic regulation and aging of satellite cell responses. Aging Cell 2009, 8(6):676-89.

41. Carlson ME, Hsu M, Conboy IM: Imbalance between pSmad3 and Notch induces CDK inhibitors in old muscle stem cells. Nature 2008, 454(7203):528-32.

42. Chien KR, Karsenty G: Longevity and lineages: toward the integrative biology of degenerative diseases in heart, muscle, and bone. Cell 2005, 120(4):533-44.

43. Tenover JL: Testosterone replacement therapy in older adult men. Int $\mathrm{J}$ Androl 1999, 22(5):300-6.

doi:10.1186/1742-4933-8-5

Cite this article as: Banerjee et al: Identification of serum biomarkers for aging and anabolic response. Immunity \& Ageing 2011 8:5. 